\title{
Évaluations par Cartes Conceptuelles à trous et apprentissage par les pairs
}

\author{
MANUELA GUISSET \\ Conseillère Pédagogique au Louvain Learning Lab de l'UCLouvain \\ Grand'Rue 54 à 1348 Louvain-la-Neuve, Belgique - manuela.guisset@uclouvain.be \\ LIESJE COERTJENS \\ Professeure à l'UCLouvain (Psychologie et Sciences de l'éducation) \\ liesje.coertjens@uclouvain.be \\ DOMINIQUE DE JAEGER \\ Professeure à l'UCLouvain (Faculté des Sciences de la Motricité) \\ dominique.dejaeger@uclouvain.be \\ GUILLAUME LOBET \\ Professeur à l'UCLouvain (Faculté des bioingénieurs) \\ guillaume.lobet@uclouvain.be \\ OLIVIER SERVAIS \\ Professeur à l'UCLouvain (École des sciences politiques et sociales) \\ olivier.servais@uclouvain.be \\ VINCENT WERTZ \\ Professeur à l'UCLouvain (École polytechnique de Louvain) \\ vincent.wertz@uclouvain.be \\ PATRICK WILLEMS \\ Professeur à l'UCLouvain (Faculté des Sciences de la Motricité) \\ patrick.willems@uclouvain.be \\ JEAN-FRANÇOIS REES \\ Professeur à l'UCLouvain (Faculté des Sciences) \\ jf.rees@uclouvain.be
}

\section{Résumé}

Cet article décrit un nouveau dispositif d'évaluation des acquis d'apprentissage basé sur des cartes conceptuelles «à trous » (CCàT) permettant également l'apprentissage par les pairs en grands auditoires durant les tests et une correction automatisée par des formulaires QCM. 
L'intérêt du dispositif est de garantir une évaluation qualitative (à haut niveau taxonomique) des apprentissages tout en facilitant la conception et la correction de ces évaluations par l'enseignant, même pour de grands groupes d'étudiants $(>500)$ et en favorisant la coopération entre étudiants en amont et pendant les évaluations.

\begin{abstract}
This article presents a new learning outcomes assessment tool based on concept maps similar to Cloze text, with peer and automated correction. The advantage of the tool is to guarantee a qualitative assessment of learning outcomes at high taxonomic level while facilitating the design and correction of these assessments by the teacher, even for large groups of students (>500) and favoring cooperation between these both before and during assessments.
\end{abstract}

\title{
Mots-clés
}

Évaluation des acquis d'apprentissage, carte conceptuelle, apprentissage par les pairs, évaluation collaborative, correction automatisée

\section{Keywords}

Learning outcomes, assessment, concept map, peer instruction, collaborative assessment, automated correction

\section{Introduction}

Testé depuis plusieurs années à l'UCLouvain, ce dispositif innovant d'évaluation des acquis d'apprentissage de grands groupes d'étudiants a fait l'objet d'une recherche approfondie afin de pouvoir le formaliser et le proposer aux enseignants de l'enseignement supérieur désireux d'améliorer leurs méthodes d'enseignement et d'évaluation. Dans cet article seront abordés le détail du dispositif, ses avantages et ses limites ainsi que des pistes pour le mettre en œuvre.

\section{Contexte}

Alors que les enseignants doivent expliciter les acquis d'apprentissage (AA) visés par leur enseignement, l'augmentation de la taille des cohortes d'étudiants dans de nombreuses filières universitaires complique l'évaluation de ces AA. Dans de nombreux cas, celle-ci recourt à des questions à choix multiples (QCM) qui permettent une automatisation de la correction des 
résultats. Dans certains cursus comptant de très grands nombres d'étudiants, la plupart, sinon la totalité, des enseignements de première année sont évalués de cette manière, ce qui peut renforcer les biais associés à ce type de questionnaires (voir au point 3) et mener à une sélection inappropriée de profils étudiants dès le début de la formation.

Par ailleurs, si l'enseignant désire mettre en place un système d'évaluation continue qualitatif appelant notamment des questions ouvertes ou de production personnelle des étudiants, il se rend vite compte que le temps consacré à l'évaluation (et à la correction) dépasse largement celui passé à enseigner ou à accompagner les étudiants dans leurs apprentissages.

C'est dans ce contexte particulier que nous avons mis en place un dispositif d'évaluation continue formative et certificative alliant les cartes conceptuelles et l'apprentissage par les pairs.

Dans la mesure où une évaluation certificative continue est relativement chronophage dans le cas d'une unité d'enseignement s'étalant sur 12 semaines, il nous paraissait également essentiel d'ajouter une dimension formative aux tests afin de garantir aux étudiants une certaine forme d'apprentissage pendant l'évaluation. Cette dimension est particulièrement prise en charge par le volet «apprentissage par les pairs » que nous développerons dans les prochaines pages.

Après un rapide état de la littérature à propos des QCM et des cartes conceptuelles, notamment en situation d'évaluation, nous présenterons nos hypothèses, l'analyse des données et les conclusions auxquelles nous sommes arrivés.

\section{L'évaluation des apprentissages : QCM et cartes conceptuelles}

\section{1. Évaluation formative et évaluation certificative}

Concept cher aux pédagogues de l'enseignement supérieur, l'alignement pédagogique (Biggs, 1996) prévoit une cohérence réfléchie entre les objectifs d'apprentissage visés par le cours, les dispositifs d'évaluation prévus pour tester ces AA et les activités pédagogiques pensées pour atteindre ces objectifs mesurés ensuite. L'évaluation formative, non contraignante, a pour objectif de tester les acquis dans un souci d'amélioration du niveau de maîtrise de la matière, de fournir un feedback à l'étudiant et de lui permettre de s'améliorer en vue de l'évaluation certificative. Celle-ci est habituellement mise en place en fin de dispositif afin de certifier la maîtrise de la matière par l'étudiant et de valider les crédits en jeu. Cette distinction implicite entre évaluation formative et certificative (ou sommative) induit l'absence de qualité formative à une évaluation certificative, puisqu'elle arrive généralement en fin d'enseignement et que les 
consultations et retours sur les «examens »sont facultatifs. Dans le cas d'une évaluation certificative continue, l'a priori est tout différent : la volonté d'apprendre du test certificatif est réelle étant donné que le dispositif pédagogique n'est pas terminé. L'étudiant a alors tout intérêt à apprendre de ses potentielles erreurs en prévision de l'évaluation suivante. Nous reviendrons sur ce point lors de la présentation du dispositif.

\subsection{Questions à choix multiples}

Les évaluations faisant appel aux questions à choix multiples sont monnaie courante dans les grands auditoires. En effet, la rapidité, voire l'automatisation de leur correction favorise leur choix parmi d'autres méthodes d'évaluation plus chronophages pour l'enseignant. Cependant, les QCM présentent aussi des inconvénients, parmi lesquels nous pouvons citer : la tentation de se focaliser sur des détails plutôt que d'évaluer la vision globale de la matière et les liens entre les concepts ; le fait de favoriser la mémorisation plutôt que le raisonnement, ainsi qu'une contraction du champ cognitif, donnant l'impression simpliste qu'une question n'a qu'une réponse unique ; le risque que les réponses fausses (leurres) ne soient mémorisées et perturbent l'apprentissage, en particulier en début de formation (Leclercq, 1986), le recours possible à la pénalisation des mauvaises réponses qui fait intervenir des variables non pertinentes pour la mesure des connaissances (Lecroart, 2016). Enfin, concevoir un QCM de qualité est une tâche lourde et difficile parce qu'elle nécessite de générer des réponses fausses mais plausibles et à renouveler régulièrement sa banque de questions.

À côté de ces inconvénients, des qualités sont associées à l'analyse automatisée des réponses : rapidité, simplicité, fiabilité et objectivité constituent des atouts importants pour des évaluations d'un grand nombre d'apprenants.

\subsection{Des cartes conceptuelles en enseignement}

Les cartes conceptuelles sont des représentations graphiques de connaissances, formées de nœuds (les concepts) reliés par des mots liens qui explicitent la nature du lien entre les concepts. Cette représentation est considérée comme étant proche de l'organisation des connaissances dans le cerveau (Novak, 2008). Aussi, créer une carte conceptuelle à partir de ses apprentissages constitue un exercice favorisant l'organisation des connaissances et leur mémorisation à long terme. Leur analyse par l'enseignant permet également d'identifier des représentations erronées des savoirs et de leurs relations chez les étudiants.

La littérature regorge d'exemples d'utilisation de cartes conceptuelles dans l'enseignement, en tant qu'outil d'apprentissage et d'évaluation (par ex. Barras et Dayer 2017 ; Delorme, Delestre 
et Pécuchet, 2004). La majorité de ces exemples concerne des dispositifs dans lesquels les cartes conceptuelles sont produites par les étudiants et évaluées une à une, par l'enseignant directement, par l'évaluation par les pairs ou par un protocole de comparaison quantitative des cartes produites par les étudiants avec celle, de référence, produite par l'enseignant ou par un expert. Cependant, très rares sont les recherches faisant part de cartes conceptuelles à compléter par les étudiants. Nous avons identifié une recherche iranienne portant sur un dispositif similaire (Hatami et al., 2016).

Dans ce contexte, nous développons un dispositif d'évaluation innovant qui permettrait de répondre aux principaux inconvénients des QCM tout en conservant ses qualités. La présente recherche a pour objectif de valider scientifiquement ces hypothèses.

\section{Un dispositif d'évaluation innovant}

\subsection{Des cartes conceptuelles à trous (CCàT)}

Concrètement, le dispositif d'évaluation par CCàT comprend trois documents :

- une carte conceptuelle portant sur une partie/la totalité de la matière, associant concepts et mots-liens dont une certaine proportion est remplacée par des trous ;

- une liste des concepts et mots liens manquants ;

- un/deux formulaires de réponse de type QCM identiques.

\subsection{Une mise en place particulière}

L'utilisation de deux formulaires de réponse vise à proposer une période de réflexion collective et de collaboration entre les étudiants : après un temps de travail individuel, les étudiants rendent leur premier formulaire de réponse, puis sont invités à discuter de leurs réponses avec leurs voisins. Chaque étudiant complète ensuite le second formulaire de réponse, identique au premier. L'évaluation finale tient compte des deux formulaires, avec une pondération fixée par l'enseignant (par exemple $80 \%$ de la note finale pour le premier questionnaire et $20 \%$ pour le second). La période de collaboration en tant que telle n'est donc pas évaluée mais donne à chaque étudiant l'opportunité d'améliorer sa note en accroissant, grâce au conflit cognitif induit par le débat entre pairs, sa compréhension globale de la matière. Elle permet aux étudiants de confronter leurs réponses et d'en débattre, permettant un apprentissage significatif durant l'évaluation.

Revenons ici sur notre distinction entre évaluation formative et certificative. Si ce dispositif innovant trouve évidemment sa place dans un dispositif pédagogique en tant qu'évaluation 
formative, c'est lorsqu'il est certificatif qu'il déploie tout son potentiel. En effet, nous avons constaté que l'étudiant apprend mieux d'une évaluation certificative continue, car elle est généralement suivie d'une autre épreuve certificative portant potentiellement sur la même matière. Dans notre dispositif, la seconde évaluation arrive moins de 15 minutes après la première. Il n'en faut pas plus pour que la motivation (Viau, 2009) certificative stimule l'apprentissage et donc le niveau de rétention à long terme du contenu. C'est en effet de manière immédiate que cet apprentissage lui sera utile, dans le cadre de la complétion du second questionnaire.

\section{Objectifs}

\subsection{Objectif général}

L'objectif principal de la recherche est de développer un outil d'évaluation associant les qualités des cartes conceptuelles à ceux de l'analyse automatisée des formulaires QCM.

L'intérêt du dispositif est de proposer une évaluation qualitative (à haut niveau taxonomique) des apprentissages tout en facilitant la conception et la correction de ces évaluations par l'enseignant, même pour de grands groupes d'étudiants (plusieurs centaines) et en favorisant la coopération entre étudiants en amont et pendant les évaluations.

\subsection{Question de recherche}

Dans cette étude, nous cherchons à savoir dans quelle mesure un dispositif d'évaluation par cartes conceptuelles à trous combine les qualités d'efficacité pour les enseignants (en termes de production et de correction) et pour les étudiants (en termes de qualité des apprentissages) afin de pouvoir être implémenté de manière large dans l'enseignement supérieur.

\subsection{Hypothèses}

Plusieurs hypothèses sont testées. Elles sont liées au processus d'apprentissage et au dispositif de carte conceptuelle et analysent :

1. Les cartes conceptuelles à trous sont capables de mesurer des acquis d'apprentissage requérant différents savoirs cognitifs ;

2. La manipulation des paramètres de la carte influence la validité, la fidélité et la capacité de discrimination du test ;

3. Une période de collaboration et de discussion entre participants permet d'améliorer les apprentissages ;

4. Une familiarité avec les cartes conceptuelles influence les résultats au test ; 
5. Les étudiants apprennent en complétant une carte conceptuelle à trous.

\section{Méthodologie de recherche}

La recherche, soutenue par le Fonds de Développement Pédagogique de l'UCLouvain rassemble plusieurs professeurs de l'Université et une conseillère pédagogique du Louvain Learning Lab. Issus des différents secteurs de l'institution (sciences et technologies, sciences de la santé et sciences humaines), les enseignants-testeurs ont réalisé de nombreux tests en situation réelle afin de valider les hypothèses présentées plus haut. Cependant, tous ces tests ne sont pas pris en compte dans cette étude, dû aux différences sensibles entre les mises en place du dispositif par chacun des enseignants.

Par ailleurs, des experts en évaluation des acquis ont été consultés : Liesje Coertjens, Mariane Frenay, Jean-Marc Braibant, Marc Romainville, Léticia Warnier, Asmaa Sadki. Le protocole d'entretien était systématiquement basé sur une présentation détaillée du dispositif analysé dans cet article puis d'un échange avec l'expert en fonction de son domaine de recherche. Leurs participations seront introduites dans la suite de cet article et ont principalement contribué à éclairer notre volet qualitatif en regard des sources identifiées dans la littérature.

Une revue de la littérature a également été effectuée au sujet des cartes conceptuelles (CC), des CC en enseignement, en enseignement supérieur, pour l'évaluation et dans le cas particulier des cartes conceptuelles à trous pour évaluer. Cet état de l'art a également contribué à enrichir le volet qualitatif de l'étude.

Voici les informations techniques concernant le volet quantitatif de notre recherche :

\subsection{Participants}

Ce sont 589 étudiants de l'UCLouvain qui ont participé à un ou plusieurs tests certificatifs par cartes conceptuelles à trous lors de quatre cours différents. Conjointement, ils ont rempli 3556 questionnaires qui ont ensuite été analysés. Les tests présentaient des caractéristiques différentes afin de pouvoir valider ou non nos différentes hypothèses. Cela n'a évidemment influencé en rien l'équité de l'évaluation pour les étudiants d'une même cohorte.

\subsection{Collecte et analyse des données}

Afin de confronter les hypothèses de la recherche, deux types de résultats sont pris en compte : premièrement, les notes obtenues par les étudiants aux différents tests, triées en fonction des contraintes qui leur ont été imposées; ensuite les réponses obtenues aux feedbacks récoltés 
auprès des étudiants après chaque évaluation. Ce feedback individuel en ligne consiste à recueillir les perceptions des étudiants à propos de la méthodologie utilisée et du dispositif CCàT. La combinaison de ces deux sources de données nous a permis de tirer des conclusions significatives par rapport à nos différentes hypothèses de travail.

Les tests analysés dans le cadre de notre étude quantitative ne concernent qu'une partie des expérimentations effectivement menées en auditoire. En effet, plusieurs enseignants ont adapté le dispositif en fonction de leur cours et de leur méthode d'évaluation annoncée. Leurs apports sont pris en compte dans la partie qualitative de notre recherche mais pas dans ce volet quantitatif.

Les tests pris en compte dans le volet quantitatif ont été menés dans quatre cours : cours de biologie animale en $1^{\text {er }}$ BAC (Biologie et Bioingénieurs), de biologie végétale en $1^{\text {er }}$ BAC (Bioingénieurs) et de physiologie générale des cellules animales en $2^{\mathrm{e}} \mathrm{BAC}$ (Biologie).

11 tests ont eu lieu en 2018 et 2019. Pour quatre d'entre eux, les étudiants ont répondu aux questionnaires sur Moodle (en salle informatique) par un ensemble de questions et réponses à menu déroulant. Pour les autres, les étudiants ont répondu aux questionnaires en auditoire, sur papier. Les questionnaires ont été corrigés automatiquement par le logiciel de correction des $\mathrm{QCM}$ «Contest ». Chacun des tests a duré entre 45 minutes et 1 heure :

- $\quad 30$ - 40 minutes pour le premier questionnaire ;

- 10 - 15 minutes d'apprentissage par les pairs ;

- 10 minutes pour le second questionnaire.

Les feedbacks récoltés l'ont été par le biais de «sondages » sur Moodle que les étudiants étaient invités à remplir pour valider leur note du test. Un taux de complétion très important a été constaté. Les questions y étaient de types fermées et ouvertes, relatives à leur perception de la passation du test, du point de vue de la complexité, de la rétention de l'information, de l'échange avec les pairs, etc. L'ensemble des questions du feedback est consultable en annexe 1.

Les données ont été analysées par certains membres de l'équipe de recherche: Guillaume Lobet, Jean-François Rees et Manuela Guisset. Elles l'ont été par «R », un logiciel libre basé sur un langage de programmation destiné aux statistiques et à la science des données.

\subsection{Expérimentations - tests du dispositif}

Nous vous présentons ici le contexte de présentation du dispositif de CCàT aux étudiants dans le cas des 11 tests analysés dans le volet quantitatif de notre étude. 
Le dispositif d'évaluation par cartes conceptuelles à trous a été combiné, dans les différents auditoires, à des dispositifs d'apprentissages variés : classes inversées favorisant la coopération et la collaboration entre étudiants pendant les phases de prise de connaissance de la matière, cours magistraux, dispositifs hybrides.

Les évaluations, toujours certificatives, prennent place, de manière continue, pendant toute la durée du cours (un quadrimestre). Les étudiants ont été évalués plusieurs fois par CCàT et chaque test est composé de trois parties distinctes, selon le dispositif explicité plus haut.

\section{Présentation et analyse des résultats}

\subsection{Les cartes conceptuelles à trous sont capables de mesurer des acquis d'apprentissage requérant différents savoirs cognitifs}

Les experts rencontrés dans le cadre de la recherche sont unanimes : d'après eux, le niveau taxonomique (taxonomie de Bloom et de Solo) atteint par les évaluations de type CCàT est au moins aussi élevé que dans le cas de QCM classiques, si ceux-ci sont conçus de manière idéale. C'est ici la capacité des cartes à mesurer les compétences de synthèse, de structuration et de lien entre les concepts qui est appréciée.

Dans la taxonomie de Bloom - voir la Figure 1 (Bloom, 1956 ; Krathwohl, 2002), alors que les QCM évaluent facilement la connaissance, la compréhension et, idéalement, l'application de la matière présentée au cours, les CCàT atteignent le niveau 4, c'est-à-dire l'analyse, le fait de mettre en relation les faits, énoncés ou questions.

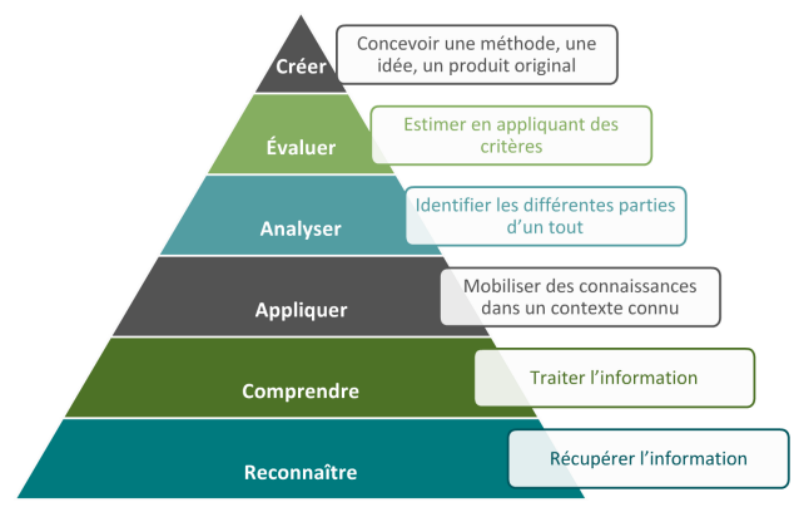

Figure 1 : Taxonomie de Bloom

Dans la taxonomie de Solo - voir la Figure 2, proposée par Biggs et Collis (1982) -, l'un de nos experts identifie le niveau relationnel (comparaison, contraste, analyse, relation, 
application) comme étant atteint alors que les QCM traditionnels se limitent à évaluer des compétences multistructurelles.

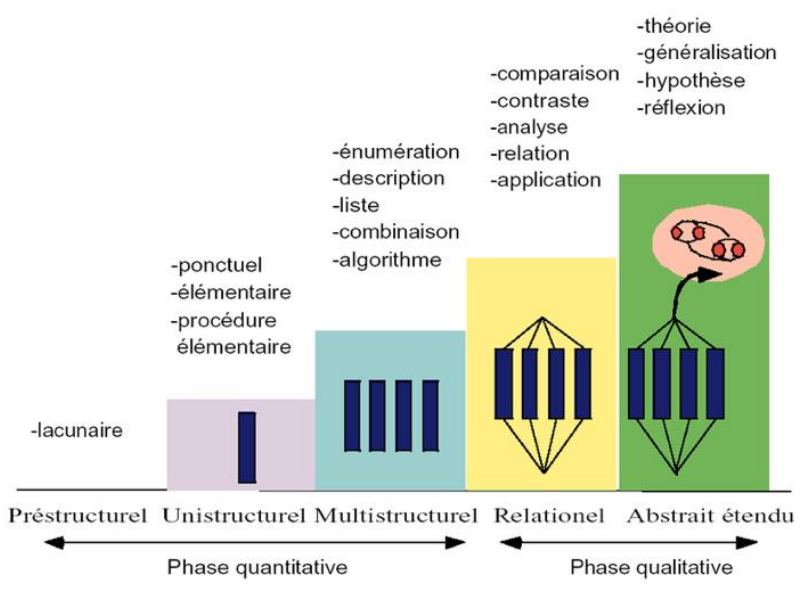

Figure 2 : Taxonomie de Solo

Par ailleurs, le volet quantitatif de notre étude relève également une corrélation parfaite entre les résultats des étudiants aux tests faisant appel aux CCàT (voir la Figure 3). La pareille est vraie pour les résultats de ces mêmes étudiants pour des activités variées, évaluées de manière continue, incluant la rédaction de textes, la réalisation de quizz en ligne et des travaux pratiques. Pour mesurer cela, les résultats des étudiants du cours de Physiologie des cellules animales (BAC2) ont été comparés aux résultats de ces mêmes étudiants lors des activités d'évaluation continue sur Moodle et lors des travaux pratiques en présentiel.

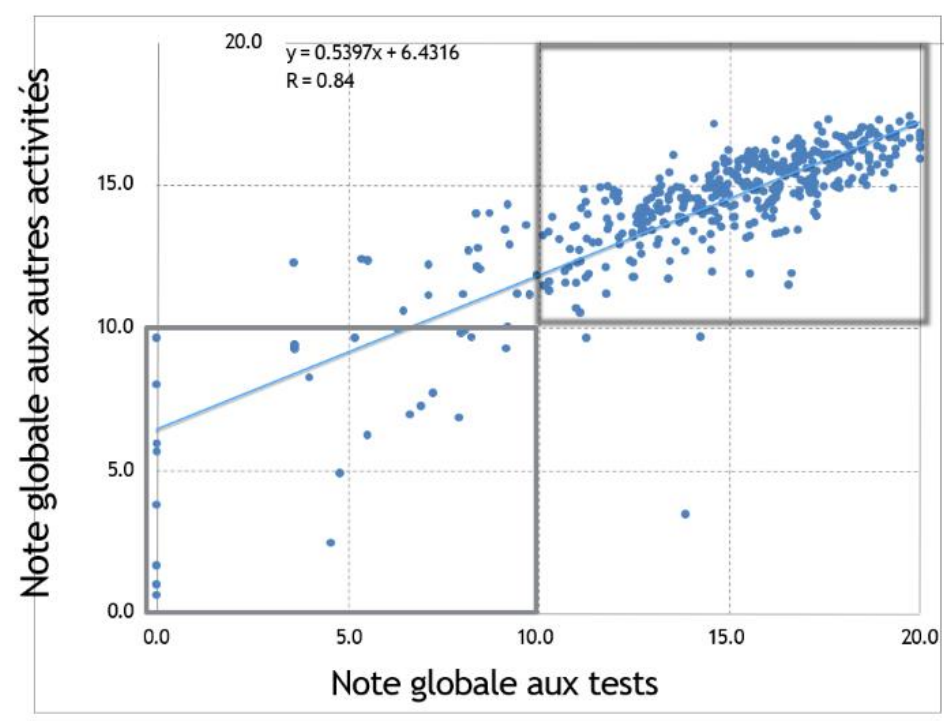

Figure 3 : Corrélation entre les résultats globaux et les résultats aux CCàT 


\subsection{La manipulation des paramètres de la carte influence la validité, la fidélité et la capacité de discrimination du test}

En interrogeant cette hypothèse, nous cherchons à savoir si le nombre de concepts présents dans la carte, le nombre de trous, le ratio entre les deux et le fait de « retourner » la présentation de la carte influencent la validité, la fidélité ou la capacité de discrimination du test.

Pour valider cette hypothèse, nous avons fait varier les valeurs d'une carte pour plusieurs groupes d'étudiants au sein d'un même auditoire. Pour garantir l'équité entre les étudiants, pour qui les tests réalisés étaient certificatifs, les modifications étaient échangées lors du test suivant.

- Faire varier le nombre de propositions de concepts à replacer

Dans une cohorte d'étudiants (Cours de Physiologie de la cellule animale en BAC2) soumis à une évaluation certificative par CCàT, la moitié du groupe (A) a reçu une carte conceptuelle avec $n$ trous et une liste de propositions composée de $n+2$ items. L'autre partie du groupe (B) a reçu la même carte mais sa liste de proposition comportant $n$-2 items ainsi qu'une proposition «Le concept n'est pas présent dans la liste » qui devait être sélectionné deux fois par les étudiants. Le même dispositif a donc été reconduit une seconde fois en prenant soin d'échanger les groupes A et B.

La question de la capacité de discrimination du test sur base du nombre d'items présents dans la liste de proposition a également été soumise à l'appréciation d'experts.

Il ressort de l'expérience menée avec les étudiants que la liste contenant $n$ - 2 items est plus difficile à gérer pour les étudiants, les résultats étant significativement (p-valeur inférieure à $0,05)$ moins élevés. Dans les questionnaires de feedback remis par les étudiants, ils ont confirmé la difficulté plus importante de cette liste incomplète.

Cette conclusion suggère que l'absence de deux concepts dans la liste force les étudiants à adopter une posture hypothético-déductive et plus seulement à travailler par élimination des concepts déjà utilisés ailleurs dans la carte.

- Faire varier le nombre de trous par rapport au nombre de concepts présents

Par ailleurs, le nombre de trous dans la carte par rapport au nombre de concepts influence la difficulté du test. Dans nos auditoires testés, la note moyenne diminue fortement lorsque l'on augmente le nombre de trous dans la carte (voir la Figure 4). C'est donc une manière simple et efficace de moduler la difficulté du test. 


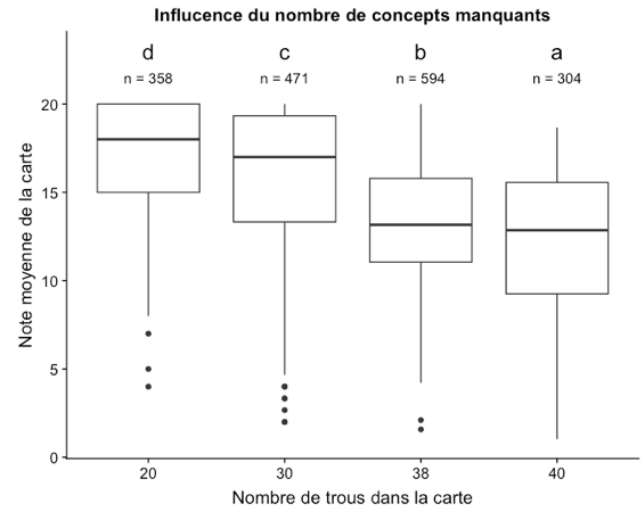

Figure 4 : Influence du nombre de concepts manquants sur la réussite

\subsection{Une période de collaboration et de discussion entre participants permet d'améliorer les apprentissages}

Afin d'évaluer la pertinence d'une période d'apprentissage collaboratif entre les périodes de complétion des deux questionnaires, nous avons comparé les résultats du Q1 (questionnaire 1) avec ceux du Q2 (questionnaire 2).

Les résultats montrent que le dispositif associant l'apprentissage par les pairs permet 95,5\% des étudiants d'améliorer leur apprentissage pendant le test (voir la Figure 5). La différence moyenne est de 3,4 points sur 20 soit une amélioration moyenne d'environ $17 \%$.

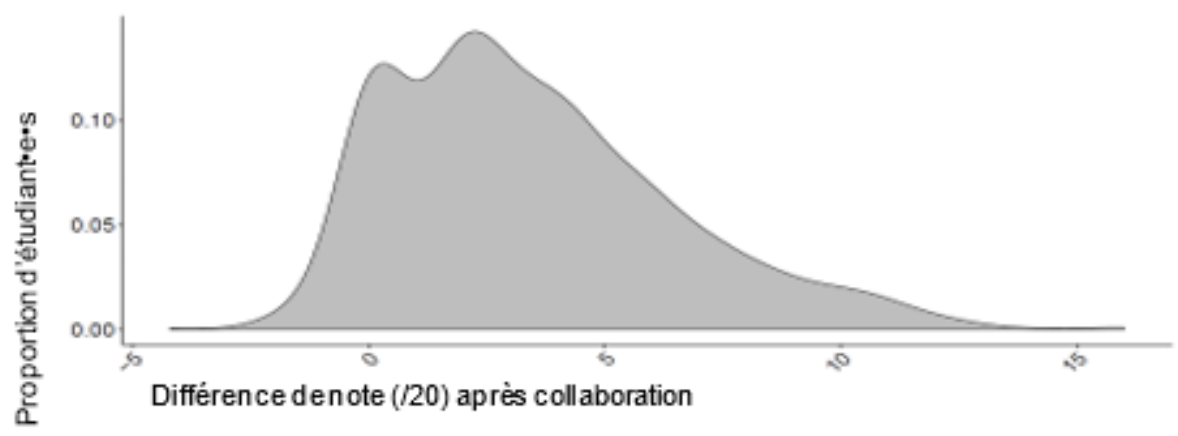

Figure 5 : Différence de note (x/20) entre Q2 et Q1

Les résultats des seconds formulaires de réponses sont presque systématiquement meilleurs que les premiers. Quand ils ne le sont pas, c'est généralement parce que l'étudiant avait déjà fait un très bon score à la première tentative.

Par contre, le nombre de personnes avec lesquelles chaque étudiant a collaboré n'influence presque pas cette différence de points (voir la Figure 6). Il n'est donc pas nécessaire de collaborer avec de nombreux collègues pour améliorer significativement sa performance au Q2. 


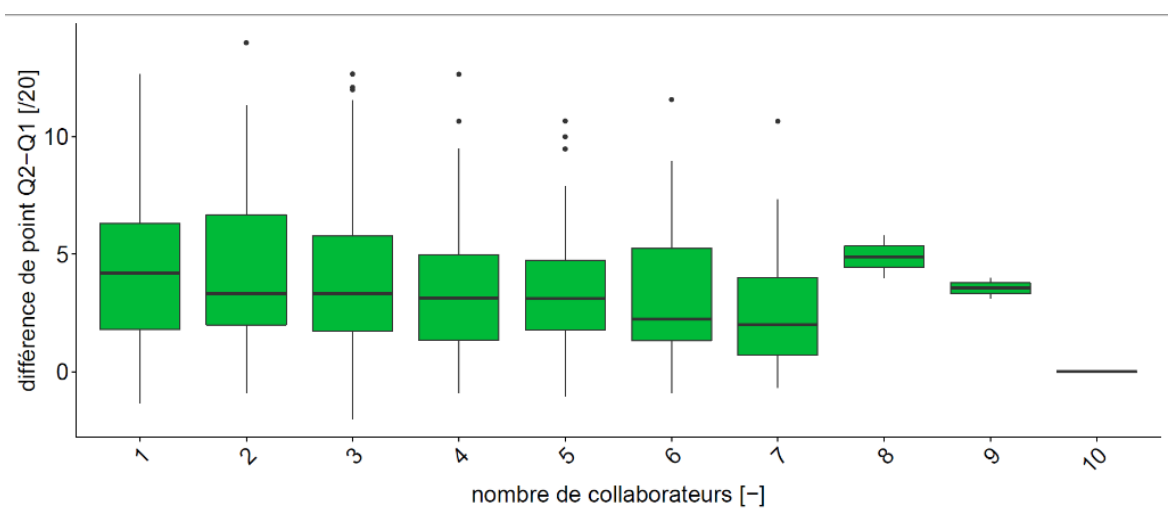

Figure 6 : Différence de note en fonction du nombre de collaborateurs

Les observations que nous avons pu mener dans les auditoires et les retours des étudiants (questionnaires de feedback) convergent dans le même sens, déjà étayé par le passé par d'autres ouvrages sur l'évaluation des acquis d'apprentissage (Gibbs et al., 2005) : la période de collaboration et d'apprentissage par les pairs entre les deux formulaires de réponse est l'occasion de créer des conflits socio-cognitifs, d'argumenter, voire de débattre des réponses des uns et des autres, plus que de copier les propositions des voisins. Nous avons assisté à de longues explications, contre-argumentations et le plus souvent consensus autour de «propositions » (au sens de Novak, 2008), de liens logiques entre deux concepts (ou plus). 64,8\% des étudiants ont rapporté avoir compris des concepts qu'ils n'avaient pas pu bien saisir au moment de l'étude ou qu'ils pensaient avoir appréhendé dans leur globalité, en lien avec le reste de la matière, alors que ce n'était pas le cas.

On remarque aussi que les étudiants collaborateurs font parfois collectivement de meilleurs résultats au second test que le meilleur d'entre eux individuellement, confirmant l'efficacité de l'intelligence collective lorsque les étudiants sont bien préparés (étude poussée comme pour un examen) pendant le test certificatif pour accroître la compréhension de la matière par les étudiants pendant une période d'évaluation.

\subsection{Une familiarité avec les cartes conceptuelles influence les résultats au test}

Pour tester cette hypothèse, nous avons comparé deux auditoires bénéficiaires du même enseignement et du même mode d'évaluation (voir la Figure 7). En amont des tests par CCàT, nous avons formé l'un des deux groupes à la lecture et à la conception autonome de cartes conceptuelles. Ces étudiants ont également bénéficié d'un «test à blanc » pour appréhender sans conséquences le dispositif d'évaluation avant le premier test certificatif. Les résultats de cette comparaison et les retours des étudiants par les feedbacks mènent au constat selon lequel la différence entre les deux auditoires est présente mais n'est pas suffisamment élevée que pour 
conclure à une réelle influence de la formation. Nous notons donc ici le caractère relativement universel de l'outil carte conceptuelle. En effet, mettre les étudiants sans préparation devant un test utilisant ces cartes n'a pas pénalisé ces étudiants.

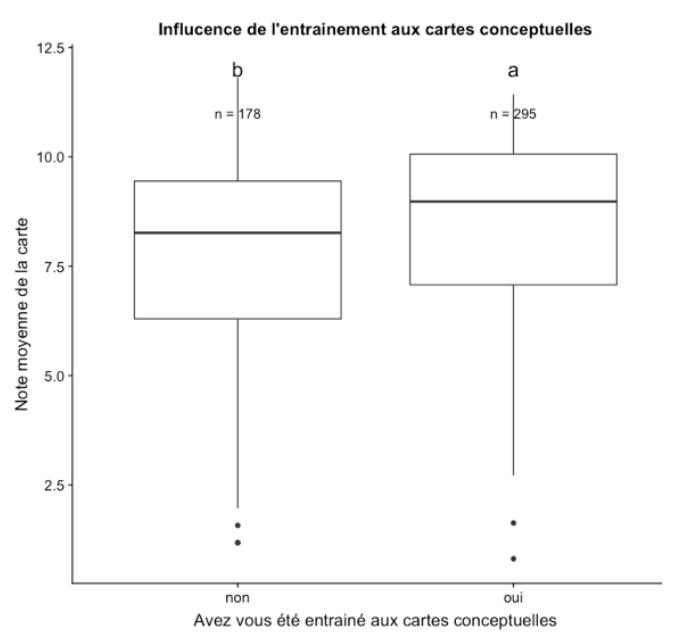

Figure 7 : Influence de la familiarité avec les cartes conceptuelles

\subsection{Les étudiants apprennent en complétant une carte conceptuelle à trous}

Comme le proposent Gibbs et Simpson (2005), les activités d'évaluation peuvent également être riches de nouveaux apprentissages. En effet, la rétroaction immédiate et personnalisée offerte par les pairs lors de la période de discussion renforce les apprentissages au moment le plus opportun, à savoir celui où la prise de connaissance du contenu est récente (étude pour le test). Il s'agit alors de faire progresser la compréhension de chacun par le biais d'argumentations et d'explications supplémentaires.

Pour mesurer cette hypothèse, lors d'un dispositif d'évaluation certificative par CCàT, les étudiants se sont vus proposer une évaluation en deux parties : un quizz de type QCM et une carte conceptuelle à trous. Les deux formats d'évaluation portaient exactement sur la même matière. La moitié de l'auditoire (A) s'est vue proposer le quizz avant la carte et l'autre moitié (B) la carte avant le quizz. Lors du test suivant, les attributions des deux groupes ont été inversées afin de tester la validité du processus. Dans les deux cas, un «groupe témoin » de deux questions QCM n'ayant pas de rapport avec les concepts présentés dans la carte (mais faisant partie de la matière à étudier) a été proposé aux étudiants.

Les résultats sont significatifs : les groupes ayant commencé par compléter la carte ont systématiquement mieux réussi le quizz que le groupe ayant commencé par le quizz (augmentation des résultats de $11 \%$ en moyenne). Les deux questions sans rapport avec la carte ont, elles, été réussies de la même manière par les deux groupes (différence de $2 \%$ ). Nous 
pouvons donc en conclure que la carte conceptuelle a aidé les étudiants à répondre aux questions du quizz et qu'ils ont appris pendant la réalisation du test.

\section{Limites et perspectives}

Les cartes conceptuelles restent des outils fortement dépendants de l'enseignant qui les conçoit. Une attention très particulière doit être apportée à la conception, qui doit être la plus objective et universelle possible afin de limiter les risques de confusion ou de réponses multiples possibles. Une relecture attentive doit être opérée, si possible par un regard extérieur, afin de repérer les incohérences, doublons, coquilles, erreurs dans le dédoublement en séries de l'évaluation, etc. Si corriger une coquille en temps réel avec un petit groupe d'étudiants évalués par un outil informatique est imaginable, aucune erreur n'est permise lors d'une évaluation en grand auditoire.

Par ailleurs, une réserve est à souligner : certains étudiants ne sont pas à l'aise avec le principe de cartes conceptuelles. Tout comme d'autres ont des difficultés avec un examen oral, une rédaction ou un QCM, de nombreux étudiants nous ont rapporté (feedbacks étudiants) être peu enclins à organiser leur pensée sous forme de carte conceptuelle ou se sentir rapidement perdus face à une carte. Une variété des formes d'évaluation tout au long du cursus de l'étudiant est donc importante. Cette limite fera l'objet d'un approfondissement dans de futures publications afin de pouvoir l'appréhender au mieux. Néanmoins, comme indiqué ci-dessus (voir 7.1.), nous avons constaté que cette limite ne se vérifie pas dans les notes obtenues, car, en moyenne, la réussite aux tests réalisés avec les CCàT est très fortement corrélée à la réussite globale des étudiants évalués par des QCM et la rédaction de travaux personnels.

Finalement, comme indiqué précédemment, la littérature scientifique, présente abondamment pour documenter l'utilisation des cartes conceptuelles (et leurs cousines les cartes mentales ou mindmap) dans l'enseignement supérieur, comme outil d'apprentissage ou d'évaluation (par production de l'étudiant), est pratiquement inexistante à propos de l'évaluation par des cartes conceptuelles à trous. Nous procédons donc ici par tâtonnement, avançant au gré de nos essais et de nos erreurs afin de définir un dispositif qui réponde le plus possible aux besoins des enseignants et des étudiants. Si les théories récentes en matière d'apprentissage nous sont absolument indispensables dans nos travaux, nous manquons cependant de points de comparaison avec d'autres expérimentations de dispositifs similaires qui auraient été menées par ailleurs. 
Les prochaines étapes de la recherche consistent notamment à l'élargissement des expérimentations du dispositif ainsi qu'à la validation de sa transférabilité dans tous les secteurs d'apprentissages de l'enseignement supérieur. Il s'agira également de développer les outils numériques nécessaire à une conception et une correction aisée des cartes impliquant, potentiellement, la complétion de celles-ci à distance.

\section{Conclusion}

Les cartes conceptuelles produites par les étudiants sont des outils reconnus et appréciés en pédagogie de l'enseignement supérieur, que ce soit en situation d'apprentissage ou d'évaluation, pour leur structure et les liens qu'elles proposent entre des concepts parfois difficiles. Les évaluations par questions à choix multiples (QCM) sont également légion, plébiscitées pour l'automatisation de leurs corrections ainsi que la possibilité de créer une banque de questions récurrentes.

Les cartes conceptuelles à trous (CCàT) combinent les avantages de ces deux outils, offrant aux enseignants une méthode d'évaluation des acquis facile à préparer et à corriger tout en garantissant un niveau taxonomique élevé des apprentissages et aux étudiants une occasion d'apprendre pendant la complétion individuelle de la carte et pendant la période d'apprentissage par les pairs proposée entre deux complétions d'une même carte.

Utilisées comme outil d'évaluation formative ou certificative, de manière ponctuelle ou continue, les cartes conceptuelles à trous permettent à l'enseignant de s'assurer que les étudiants perçoivent la matière dans sa globalité, sont capables de faire et de caractériser des liens entre des concepts vus précédemment et de les rattacher aux connaissances préétablies. S'appuyant sur les théories actuelles de l'apprentissage, les CCàT proposent l'évaluation de compétences et de connaissance d'un niveau taxonomique (Bloom revisité, Solo) important en permettant la mise en relation entre des concepts qui doivent être auparavant maitrisés. Cela sous-tend la nécessité, de la part de l'étudiant, de maîtriser la matière dans sa globalité, tant en ce qui concerne les détails que les concepts et les liens entre ceux-ci.

Par ailleurs, la préparation, vivement conseillée, de l'évaluation par l'étudiant à l'aide de cartes conceptuelles qu'il produit lui-même est gage de capacité de synthèse et de restitution.

L'automatisation de la correction, à l'aide des outils traditionnels de prise en charge des QCM (scanners optiques par ex.), garantit une rapidité, une objectivité et une aisance non négligeables 
et permet à l'enseignant de libérer du temps pour ses missions d'enseignement, de recherche et de service.

\section{Références bibliographiques}

Barras, H. et Dayer, É. (2017). Comment faire appel à une carte conceptuelle pour évaluer les apprentissages ? (chap. 13). Dans V. Roulin (dir.), Comment évaluer les apprentissages dans l'enseignement supérieur professionnalisant : Regards d'enseignants (p. 183-196). Louvain-la-Neuve : De Boeck Supérieur.

Biggs, J. (1996). Enhancing teaching through constructive alignement. Higher Education, 32, 347-364.

Biggs, J. et Collis, K. (1982). Evaluating the quality of learning: The SOLO taxonomy. New York: Academic Press.

Bloom, B. (1956). Taxonomy of educational objectives. Handbook 1: Cognitive domain. New York: David McKay Company Inc.

Bouvy, T. et Warnier, L. (2011). Document de synthèse sur les QCM [document inédit]. Université catholique de Louvain, Louvain-la-Neuve.

Cronbach, L. et Meehl, P. (1955). Construct validity in psychological tests. Psychological Bulletin, 52(4), 281-302 (doi: 10.1037/h0040957).

Daley, B. J. et Torre, D. M. (2010). Concept maps in medical education: An analytical literature review. Medical Education, 44, 440-448.

Davies, M. (2011). Concept mapping, mind mapping and argument mapping: What are the differences and do they matter? Higher Education, 62, 279-301.

Delorme, F., Delestre, N. et Pécuchet, J.-P. (2004). Évaluer l'apprenant à l'aide de cartes conceptuelles. Technologies de l'information et de la connaissance dans l'enseignement supérieur et l'industrie. Compiègne : Université de Technologie de Compiègne, p. 25-31.

Gibbs, G. et Simpson, C. (2005). Conditions under which assessment supports students' learning. Learning and Teaching in Higher Education, 1, 3-31.

Hatami, J., Farokhnia, M. et Hasanzadeh, M. (2016). Select-and-fill-in concept maps as an evaluation tool. Science Classrooms, 635, 169-180.

Krathwohl, D. R. (2002). A revision of Bloom's taxonomy: an overview. Theory Into Practice, 41(4), 212-218.

Leclercq, D. (1986). La conception des questions à choix multiple. Bruxelles : Labor.

Lecroart, I. (2016). L'évaluation certificative par QCM à l'université. La pénalisation des mauvaises réponses est-elle associée à une meilleure discrimination des étudiants? Louvain-la-Neuve: Faculté de psychologie et des sciences de l'éducation, Université catholique de Louvain.

Novak, J. D. (2008). The theory underlying concept maps and how to construct them. Technical report, Institute for Human and Machine Cognition, Stanford University.

Romainville, M., Goasdoué, R. et Vantourout, M. (2013). Évaluation et enseignement supérieur. Louvain-la-Neuve : De Boeck Supérieur. 
Svinicki, M. et McKeachie, W. J. (2014). McKeachie's teaching tips (14 éd.). Belmont, CA: Wadsworth.

Viau, R. (2009). La motivation à apprendre en milieu scolaire. Montréal : Pearson-ERPI.

Zvauya, R., Pu-randare, S., Young, N. et Pallan, M. (2017). The use of mind maps as an assessment tool in a problem-based learning course. Creative Education, 8, 1782-1793. 


\section{ANNEXE 1}

\section{Questions des feedbacks des étudiants :}

\begin{tabular}{|c|c|}
\hline $\mathrm{fb} 1$ & Avez-vous déjà produit une carte conceptuelle vous-même? \\
\hline $\mathrm{fb} 2$ & Avez-vous déjà été évalué·e par carte conceptuelle par le passé ? \\
\hline fb3 & Vous êtes-vous préparé-e à ce test à l'aide de cartes conceptuelles? \\
\hline $\mathrm{fb} 4$ & $\begin{array}{l}\text { Avez-vous consulté les ressources proposées dans le cours Moodle pour vous } \\
\text { familiariser avec les cartes conceptuelles? }\end{array}$ \\
\hline fb5 & Avez-vous téléchargé et installé le logiciel CmapTools sur votre ordinateur? \\
\hline fb6 & $\begin{array}{l}\text { Comment avez-vous procédé pour compléter la carte conceptuelle lors de la première } \\
\text { tentative? (plusieurs réponses possibles) }\end{array}$ \\
\hline $\mathrm{fb} 7$ & La période de discussion avec les autres étudiants était-elle utile? \\
\hline $\mathrm{fb} 8$ & Avez-vous appris de nouvelles choses pendant la période de discussion? \\
\hline $\mathrm{fb} 9$ & Pensez-vous avoir fait un meilleur résultat au second test? \\
\hline $\mathrm{fb} 10$ & $\begin{array}{l}\text { Pour vous, la carte conceptuelle était-elle difficile à compléter ? (1: très facile à } 10 \text { : } \\
\text { très difficile) }\end{array}$ \\
\hline $\mathrm{fb} 11$ & Le test était-il réalisable dans le temps imparti ? \\
\hline $\mathrm{fb} 12$ & Pensez-vous être à l'aise avec le principe de cartes conceptuelles ? \\
\hline $\mathrm{fb} 13$ & $\begin{array}{l}\text { Pour vous, l'évaluation par carte conceptuelle à trous (CCàT) permet-elle d'évaluer } \\
\text { différemment qu'un QCM ordinaire? }\end{array}$ \\
\hline $\mathrm{fb} 14$ & Le fait d'avoir 2 concepts absents de la liste de propositions ... \\
\hline $\mathrm{fb} 15$ & A propos des deux concepts absents de la liste de propositions : \\
\hline $\mathrm{fb} 16$ & Comment avez-vous procédé pour trouver les mots-liens manquants ? \\
\hline $\mathrm{fb} 17$ & Devoir justifier le choix des mots-liens m'a paru (facile-difficile) \\
\hline $\mathrm{fb} 18$ & $\begin{array}{l}\text { Devoir justifier le choix des mots-liens pour la qualité de l'évaluation (embrouille- } \\
\text { meilleure éval) }\end{array}$ \\
\hline $\mathrm{fb} 19$ & La période de formation aux cartes conceptuelles était-elle utile? \\
\hline $\mathrm{fb} 20$ & Vous avez réalisé votre propre carte conceptuelle ce jeudi ... \\
\hline $\mathrm{fb} 21$ & $\begin{array}{l}\text { Après cette séance de formation, vous sentez-vous à l'aise pour préparer les futurs } \\
\text { tests à l'aide de cartes conceptuelles }\end{array}$ \\
\hline
\end{tabular}




\begin{tabular}{|l|l|}
\hline $\mathrm{fb} 22$ & $\begin{array}{l}\text { Le fait que les cartes n'étaient pas présentées de la même manière en fonction des } \\
\text { séries ... }\end{array}$ \\
\hline $\mathrm{fb} 23$ & Les deux schémas supplémentaires ... \\
\hline $\mathrm{fb} 24$ & Dans ma liste de propositions de concepts/mots-liens, il y avait : \\
\hline $\mathrm{fb} 25$ & Si (et seulement SI) vous aviez PLUS de propositions que de trous \\
\hline $\mathrm{fb} 26$ & Si (et seulement SI) vous aviez MOINS de propositions que de trous \\
\hline $\mathrm{fb} 27$ & Je pense que \\
\hline $\mathrm{fb} 28$ & $\begin{array}{l}\text { (Le cas échéant) Le fait d'avoir déjà été évalué.e par carte conceptuelle vous a-t-il } \\
\text { aidépondre à celle-ci ? }\end{array}$ \\
\hline $\mathrm{fb} 29$ & Cette carte conceptuelle-ci était présentée verticalement ... \\
\hline $\mathrm{fb} 30$ & Le fait de connaitre la liste de concept à l'avance ... (plusieurs réponses possibles) \\
\hline $\mathrm{fb} 31$ & $\begin{array}{l}\text { Sur le schéma ci-dessus, dans quelle partie de la salle Darwin vous situiez-vous } \\
\text { durant le test ? }\end{array}$ \\
\hline $\mathrm{fb} 32$ & Autre chose à rajouter ? \\
\hline $\mathrm{fb} 33$ & Par quelle partie du questionnaire avez-vous commencé ? \\
\hline $\mathrm{fb} 34$ & Pour vous, le quizz était-il difficile à compléter ? (1 : très facile à 10 : très difficile) \\
\hline $\mathrm{fb} 35$ & A quelle partie du test avez-vous eu le plus facile à répondre ? \\
\hline $\mathrm{fb} 36$ & Avoir commencé par la carte/le quizz m'a aidé pour répondre au quizz/à la carte \\
\hline
\end{tabular}

2. Annexe 2 : Exemple de carte conceptuelle à trous

3. Annexe 3 : Liste des concepts à replacer dans la carte 


\section{Groupe B}

Tableau 1

11-Cis- Rétinal

All-Trans-Rétinal

Axones

Bâtonnets

Canaux Ca2+ voltage- dépendants

Canaux $\mathrm{Na}+$ chimio-dépendants

Capillaires sanguins

Cellules bipolaires

Cellules ganglionnaires

Cellules horizontales

Cônes

Faible convergence

Forte convergence vers

Glutamate

GMPc

Guanylate cyclase

Humeur vitrée

Le concept n'est pas présent

Métabolise(nt)

Métabolisme cellulaire

Partie protéique

Phosphodiestérase

Photorécepteurs

Pigments visuels

Porphyropsines

Potentiel d'action

Potentiel graduel

Potentiel récepteur

Rédui(sen)t la libération de

Rhodopsine

Sclérotique

Sensibilité spectrale

Vision diurne

Vision nocturne 